\title{
Effect of Pain and Hip Muscle Strength and Flexibility on Functional Status of Females with Patellofemoral Pain Syndrome
}

\author{
Hira Jabeen ${ }^{1}$, Muhammad Salman Bashir ${ }^{2}$, Sarah Ehsan ${ }^{3}$ \\ ${ }^{1}$ Senior Lecturer, Azra Naheed Medical College, Department of Physical Therapy, Main Raiwind Road,, Lahore \\ ${ }^{2}$ Head of department/Associate Professor, Department of physical therapy and rehabilitation sciences, FHAS,ICBS, Lahore \\ ${ }^{3}$ Senior lecturer, Azra Naheed Medical College, Department of Physical Therapy, Main Raiwind Road,, Lahore
}

\begin{abstract}
The objective of this study was to determine the effect of pain and hip muscle strength and flexibility on functional status of females with the Patellofemoral pain syndrome. This cross sectional study was conducted at Chaudhary Mohammad Akram hospital, Sports and Spine center and Ehsan rehabilitation center on a sample of 40 females. Non-probability, purposive sampling was used. Kujala score was used to measure the functional status of females with PFPS. Pain was measured through Visual analogue scale (VAS) and hip muscle strength and flexibility were tested through manual muscle testing (MMT) and special tests respectively. Linear regression was used to assess the effect of pain, muscle strength and flexibility on functional status using Kujala score as dependent variable. Pain explained $37.6 \%$ variation in the functional status of females with patellofemoral pain syndrome. Prediction of the functional status of females with PFPS by pain along with gluteus maximus strength was statistically significant ( $p=.000)$. The study concludes that pain in unilateral squat and strength of hip extensors are better predictors of functional status in females with patellofemoral pain syndrome. However, Hip muscle flexibility is not a good predictor of functional status.
\end{abstract}

Keywords: Patellofemoral pain syndrome, Patellofemoral joint, females, hip, knee, muscle weakness

\section{Introduction}

\subsection{Overview}

Patello femoral pain syndrome(PFPS) is described as pain on the anterior aspect of knee or persistent pain behind the patella that is increased with repetitive knee activities like jumping, running, ascending or descending stairs and with prolonged sitting as well.[1]There can be several reasons for anterior knee pain e.g. Osgood-Schlatter disease, Plica syndrome, prepatellar bursitis, pes anserine bursitis, quadriceps tendinopathy, patellar stress fractures and PFPS. The most common cause for anterior knee pain amongst all these is PFPS.[2]

PFPS is the most common diagnosis for the young individuals who complain of knee pain. Females are at a greater risk for developing PFPS. The first step towards prevention of this syndrome is identification of possible risk factors.[3] PFPS has a tendency to become chronic particularly in physically active individuals.(6)

PFPS effects 1 out of every 4 athletes, $70 \%$ of which are 16 to 25 years of age.[4]Patello femoral joint is subjected to multiple forces with increased joint compression upon knee flexion. The complexity of forces on patello femoral joint makes it somewhat difficult to treat. PFPS can be caused by intrinsic and extrinsic, modifiable and non-modifiable risk factors which should be addressed in order to prevent the disorder. The suggested modifiable risk factors include, quadriceps weakness (particularly VastusMedialisObliqus VMO), tightness of hamstring, iliopsoas and gastrosoleous muscles, hip muscles dysfunction, foot over pronation, tightness of the iliotibial band, generalized joint laxity, limb length discrepancy, patellar mal-alignment and hyper mobility. The treatment approach used must be in consideration with these risk factors in order to get better outcomes and prevent recurrence and chronicity.[5]

PFPS has detrimental effects on physical activity with an early onset of osteoarthritis of the knee. The effective preventive strategies will reduce the occurrence of PFPS as well as the resulting osteoarthritis. Some prospective cohort investigations have indicated decreased quadriceps flexibility, shortened reflex time of the vastusmedialis oblique, reduction of vertical jump performance, increased medial patellar mobility, increased medial tibialintercondylar distance, and increased quadriceps strength as the causative factors for patello femoral pain syndrome. Some factors are non-modifiable like medial tibialintercondylar distance, and thus can "etbe avoided to prevent PFPS. [1]

Altered kinetics and kinematics resulting from muscular imbalance around hip and knee have been attributed to be the modifiable risk factors of PFPS. Altered kinetics and kinematics predispose the patello femoral joint to increased loads that can culminate in PFPS. Abnormal patellar tracking can result from quadriceps imbalance leading to anterior knee pain. [1]

People with PFPS as compared to those without PFPS showed weak hip abductors, knee flexors and knee extensors strength as well as lower vertical ground reaction forces, knee extension moment and hip external rotation moment. Female gender, and reduced lower extremity strength are the most important risk factors. Internal rotation of femur results in excessive contact pressure on lateral facet of patella. [1] 


\section{International Journal of Science and Research (IJSR) \\ ISSN (Online): 2319-7064 \\ Index Copernicus Value (2013): 6.14 | Impact Factor (2015): 6.391}

There is no established relationship between an excessive Qangle and development of PFPS. [6]

Foot posture may be an important factor in causing anterior or retropatellar pain, navicular drop, navicular drift and dorsiflexion have been found to be linked with PFPS.[7]VMO and VastusLateralis (VL) play important role in dynamic stabilization of patella so the weakness of either can lead to mal-tracking of patella. Weakness of VMO leads to lateral tracking of patella which results in PFPS. [2] Tightness of Rectus femoris can also cause PFPS by increasing lateral contact forces on patello femoral joint and altering kinetics and kinematics of knee. People with PFPS are also found with weak hamstrings. [1]

Reduced and delayed gluteus medius and VMO activation is found in individuals with PFPS during stair climbing.[8]Tightness of IT has also been found to be an important risk factor. IT band has anatomical connections with lateral patellar retinaculum and thus increases lateral forces on patella during knee flexion. [1]

Patellar mal-tracking does play some role in developing PFPS. However individuals with PFPS do not always present with patellar mal tracking.[9]

Hip strengthening, quadriceps strengthening (particularly VMO), patellar taping, patellar and knee bracing and foot orthosis have been suggested as treatment options for PFPS. [10]

\subsection{Objective}

To determine the effect of pain and hip muscle strength and flexibility on the functional status in females with the Patellofemoral pain syndrome.

\subsection{Rationale}

This study was done to determine whether pain, hip muscle strength and flexibility explain the alteration in functional status of females with PFPS in current setting. Identification of these relationships would be helpful in effectively understanding the pathology and devising appropriate treatment plans that ensures best outcomes and also helps clinicians to develop preventive measures for PFPS.

\subsection{Operational Definitions}

\subsubsection{Pain}

For pain assessment of Patellofemoral syndrome the unilateral leg squat test was conducted on the patients to provoke the pain. This test was chosen for analysis because this task specifically targets the gluteus medius, gluteus maximus and hip lateral rotator muscles and it also stresses the Patellofemoral joint. [19] Subjects of the study were asked to perform a partial unilateral squat to approximately $60^{\circ}$ of knee flexion (on the painful side). For balance purpose only two-finger support was allowed. After performing this task, subject was asked to mark a point on VAS according to pain level she perceived. Then VAS scores were calculated by measuring the length on the VAS scale in mm. The VAS was a $100-\mathrm{mm}$ horizontal line with left and right anchors representing „no pain ${ }^{\text {ee }}$ and „worst imaginable pain ${ }^{\text {ee }}$,respectively. [11]

\subsubsection{Hip muscle strength}

Strength assessment of gluteus medius, gluteus maximus and hip lateral rotators were done on the involved side using the manual muscle testing (MMT) procedures. [14]

\subsubsection{Gluteus Medius}

To test the gluteus medius, patients were positioned on a plinth in side lying with involved leg up. The lower leg was slightly flexed to provide trunk stability. Some manual assistance to pelvis is also given by the examiner to stabilize it. The upper leg was positioned in slight hip abduction, extension and lateral rotation, and subjects were told to ,hold the position and don "et let me move you ${ }^{\text {ee }}$ The examiner applied resistance in the direction of hip adduction and flexion. Strength was assessed on MMT scale with 0 considered as no palpable or observable muscle contraction and 5 present patient moves against gravity and maximal resistance over the full $\mathrm{ROM}$

\subsubsection{Gluteus Maximus strength}

Gluteus maximus strength was assessed with subjects in the prone lying position. The involved knee was flexed to90 and the involved hip was extended without allowing the subject to extend the trunk. The pelvis was stabilized manually by the examiner. The subject was asked to hold the position while force was applied perpendicular to the limb in the direction of hip flexion.

\subsubsection{Lateral rotator strength}

To test lateral rotator strength, subjects assumed a seated position with the hips and knees flexed to $90^{\circ}$. A small towel was placed under the knee to align the knee joint center with the hip joint center. The subject"s involved limb was placed in lateral rotation while the examiner stabilized the femur. The subject was asked to hold the position while the examiner applied force perpendicular to the limb in the direction of medial rotation.

\subsubsection{Hip muscle flexibility}

\subsubsection{Iliotibial band length}

Modified Ober"es test was used to assess the Illiotibial band length. Intrarater reliability of modified Ober's test was calculated to be 0.91. [15] The procedure involves positioning the subject in side lying with the involved side up. Lower leg is flexed with hip and knee flexed in a 90degree angle. The examiner placed the testknee in extension, fully abducts the lower extremity that needs to be tested, then allows the force of gravity to adduct the extremity until the hip cannot adduct any further

\subsubsection{Hamstring length (Passive SLR)}

The patient was supine with hips and knees in $90^{\circ}$. The examiner passively straightens the lower leg. Normal length of the hamstring muscle is $90^{\circ}$ hip position with straight knee. Less than $80^{\circ}$ is referred as loss of hamstring extensibility.

\subsubsection{Rectus femoris length}




\section{International Journal of Science and Research (IJSR) \\ ISSN (Online): 2319-7064 \\ Index Copernicus Value (2013): 6.14 | Impact Factor (2015): 6.391}

The test was performed with patient in supine with both knees maximally flexed, using both arms. This ensures that the lumbar spine is flexed and flat on the table and avoids a posterior tilt of the pelvis. The patient then lowered the tested limb toward the table. During the test the contra lateral hip was held in maximal flexion. The length of the iliopsoas was measured by the angle of the hip flexion.

\subsubsection{Functional status}

For assessing the functional status of females with PFPS an international tool was used called Anterior Knee Pain Questionnaire (AKPQ) developed by Kujala et al. [12]. It is a self-reporting knee pain questionnaire with 13 multiple choice questions were present to evaluate the functional limitations caused by patellofemoral pain. These questions address the issues of pain, swelling, patellar subluxation, quadriceps atrophy, knee flexion deficiency. It documents the response of patient on six physical activities that are walking, stairs, squatting, running, jumping and sitting. The composite score ranges from 0 to 100 , with 100 indicating no functional limitation. Scores of 70 are considered to represent moderate disability. The Kujala questionnaire has demonstrated a high test-retest reliability ICC $=.86-.94$. [13]

\subsection{Materials and methods}

\subsubsection{Study design}

This study was an observational, cross-sectional study

\subsubsection{Setting}

Data was collected from females of Chaudhary Muhammad Akram hospital, Ehsan Rehabilitation center and Sports and Spine Center Lahore. The data was compiled at Riphah College of Rehabilitation Sciences, Lahore

\subsubsection{Study population}

Young females having patellofemoral pain syndrome in one or both knees.

\subsubsection{Duration of study}

4 months after the approval from ethical review board.

\subsubsection{Sample size}

The Sample of 40 females was selected using 6\% prevalence and $9 \%$ level of significance. Following formula was used for sample size calculation:

$$
\mathrm{n}=\frac{z_{1 \cdot x / s}^{2} \mathrm{P}(1-\mathrm{P})}{\mathrm{d}^{2}}
$$

\subsubsection{Eligibility}

\subsubsection{Inclusion criteria}

Females between 18 to 40 years of age, having retropatellar pain for at least 1 month.

\subsubsection{Exclusion criteria}

Patients with any history of knee ligament, tendon or cartilage injury, traumatic dislocation of patella, knee surgery and systemic disease (Rheumatoid arthritis,gout, pseudo gout, and autoimmune diseases), neuropathic arthropathies and infectious joint diseases were excluded from the study.

\subsubsection{Data collection}

Fourty females fulfilling the inclusion criteria were selected using purposive sampling. The identity of the researcher was kept anonymous. All measures were collected during a single session.After taking informed consent patients were requested to fill in their activity score to assess their level of work and sport activities.Unilateral squat test was performed on the effected side and the level of pain was recorded on the visual analogue scale (VAS). Muscle strength was assessed using the manual muscle testing and flexibity tests for Illiotibial band, hamstrings and rectus femoris were done using modified Ober's test, passive SLR and Thomas test respectively. Functional status of knee was assessed using the anterior knee pain questionnaire (Kujala score) which is a self-administered questionnaire. Each session, including both questionnaire and assessment of strength and flexibility took 30 minutes to complete for each patient.

\subsubsection{Ethical Consideration}

Study was conducted after taking approval from the ethical committees of Chaudhary Muhammad Akram hospital, Ehsan Rehab and sports and spine center.Written informed consent was obtained from all subjects and the rights of privacy of the personal details of the subjects were protected.

\subsubsection{Statistical Procedure}

Data was analyzed using SPSS v. 20.00. Mean \pm SD was calculated for quantitative variables and frequencies, percentages and bar charts were used for categorical data. Linear regression was used to assess the effect of pain on functional status using Kujala score as dependent variable and pain as independent. Multiple hierarchical regression was used to analyze the effect of pain alone and pain along with gluteus maximus strength on the functional status. Muscle flexibility and gluteus medius, minimus and hip lateral rotator strengths were not included in hierarchical regression as they did not have significant correlation with functional status individually. $\mathrm{P}$ value $\leq .05$ was considered significant.

\section{Results}

The average age of the females participated in the study (mean \pm standard deviation [SD]) was 28 44 years. Mean

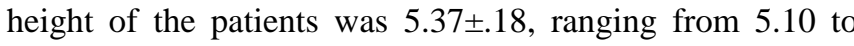
5.37 feet. Mean weight of the patients was $56.25 \pm 6.34$, ranging from 47 to 72 kilos. The mean BMI of the patients was 21.52 \pm 1.95 , with a minimum BMI of 17.70 and maximum of $26.40 \mathrm{~kg} / \mathrm{m}^{2}$.

Table 1: descriptive statistics of muscle flexibility tests

\begin{tabular}{|c|l|c|c|}
\hline \multicolumn{2}{|c|}{} & Frequency & Percentage (\%) \\
\hline $\begin{array}{c}\text { Rectus femoris/ } \\
\text { Thomas test }\end{array}$ & Positive & 28 & 70 \\
\cline { 2 - 4 } & Negative & 12 & 30 \\
\hline $\begin{array}{c}\text { Ober"s Test for } \\
\text { IT band }\end{array}$ & Positive & 31 & 77.5 \\
\cline { 2 - 4 } & Negative & 9 & 22.5 \\
\hline $\begin{array}{c}\text { Passive SLR for } \\
\text { hamstrings }\end{array}$ & Positive & 27 & 67.5 \\
\cline { 2 - 4 } & Negative & 13 & 32.5 \\
\hline
\end{tabular}




\section{International Journal of Science and Research (IJSR) \\ ISSN (Online): 2319-7064}

Index Copernicus Value (2013): 6.14 | Impact Factor (2015): 6.391

Table 2: Descriptive statistics for muscle strength

\begin{tabular}{|c|c|c|c|}
\hline & Mean \pm SD & Minimum & Maximum \\
\hline Gluteus mediusminimus & $3.85 \pm .770$ & 2 & 5 \\
\hline Gluteus maximus & $4.13 \pm .516$ & 3 & 5 \\
\hline Lahteral rotators of hip & $4.15 \pm .533$ & 3 & 5 \\
\hline
\end{tabular}

Table 3: level of pain on VAS after unilateral squat test

\begin{tabular}{|c|c|c|c|}
\hline & Mean \pm SD & Minimum & Maximum \\
\hline Pain & $\mathbf{6 . 3 3} \pm \mathbf{1 . 7 4 5}$ & $\mathbf{4}$ & $\mathbf{1 0}$ \\
\hline
\end{tabular}

Table 4: Anterior knee pain/Kujala Score

\begin{tabular}{|c|c|c|c|}
\hline & Mean \pm SD & Minimum & Maximum \\
\hline Total mean score & $63.85 \pm 7.116$ & 49 & 81 \\
\hline
\end{tabular}

Table 5: Effect of pain on functional status

\begin{tabular}{|c|l|l|c|c|}
\hline Predictor & $\mathrm{R}$ & $\mathrm{R}^{2}$ & Adjusted $\mathrm{R}^{2}$ & $\begin{array}{c}\text { SE** of } \\
\text { estimate }\end{array}$ \\
\hline Pain & .613 & 376 & .360 & 5.694 \\
\hline
\end{tabular}

$* \mathrm{P}$ value at or less than 0.05

**Standard Error

Table 6: Effect of hip muscle strength on functional status of females with patellofemoral pain syndrome

\begin{tabular}{|c|c|c|c|c|}
\hline Predictor & $\mathrm{R}$ & $\mathrm{R}^{2}$ & $\begin{array}{c}\text { Adjusted } \\
\mathrm{R}^{2}\end{array}$ & $\begin{array}{c}\text { SE** of } \\
\text { estimate }\end{array}$ \\
\hline Gluteus medius/minimus & .178 & .032 & .006 & 7.098 \\
\hline Gluteus maximus strength & .355 & .126 & .103 & 6.741 \\
\hline Hip lateral rotator strength & .148 & .022 & -.004 & 7.130 \\
\hline
\end{tabular}

The strength of gluteus medius and minimus explained 3.2\% variation and strength of hip lateral rotators explained 2.2\% variation in functional status of females with PFPS. The Strengths of gluteus medius, minimus and hip lateral rotators both were poor predictors of functional status of females with PFPS ( $p=.271$ and .362 respectively). The strength of gluteus maximus explained $12.6 \%$ variation in functional status and was a better predictor of functional status in females with PFPS $(\mathrm{p}=0.025)$

Table 7: effect of hip muscle flexibility on functional status of females with PFPS

\begin{tabular}{|c|c|c|c|c|}
\hline Predictor & $\mathrm{R}$ & $\mathrm{R}^{2}$ & $\begin{array}{c}\text { Adjusted } \\
\mathrm{R}^{2}\end{array}$ & $\begin{array}{c}\text { SE** of } \\
\text { estimate }\end{array}$ \\
\hline Rectus femoris flexibility & .068 & .005 & -.022 & 7.193 \\
\hline TFL and IT band flexibility & .131 & .017 & -.009 & 7.148 \\
\hline Hamstrings flexibility & .197 & .039 & .014 & 7.068 \\
\hline
\end{tabular}

The flexibility of rectus femoris, TFL \& IT band and Hamstrings explains $0.5 \%, 1.7 \%$ and $1.4 \%$ variation in functional status of females with PFPS which is not considered a meaningful amount of variance. Prediction of the functional status of females with PFPS by flexibility of Rectus femoris, TFL \& IT band and Hamstrings was statistically insignificant i.e. $\mathrm{p}=.675, \mathrm{p}=0.421$ and $\mathrm{p}=.223$ respectively.

Table 8: Effect of pain and gluteus maximus strength both on functional status of females with PFPS

\begin{tabular}{|c|c|c|c|c|}
\hline Predictor & $R$ & $R^{2}$ & Adjusted $R^{2}$ & SE** of estimate \\
\hline $1^{\mathrm{a}}$ & .613 & .376 & $22.911(1,38)$ & .000 \\
\hline $2^{\mathrm{b}}$ & .620 & .384 & $11.553(2,37)$ & .000 \\
\hline
\end{tabular}

Key terms:

$\mathbf{1}^{\mathrm{a}}=$ predictors (Pain)

$2^{b}=$ predictors (Pain and Gluteus maximus strength)

The multiple hierarchical regression analysis showed that pain alone and pain with gluteus maximus strength together were good predictors of functional status in females with PFPS i.e. $p=.000$. The pain alone explained $61.3 \%$ while pain along with gluteus maximus strength explained 62\% variation in functional status of females with PFPS.

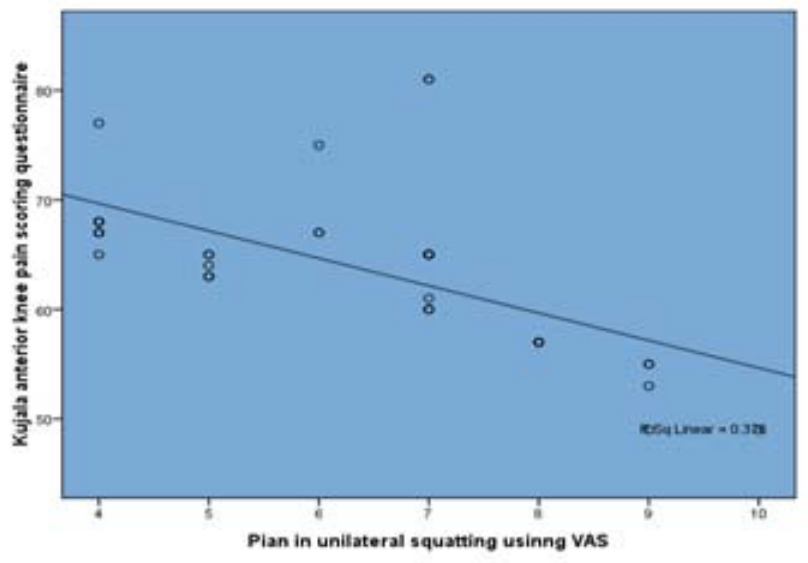

Figure 1: Scatter Plot for effect of pain on functional status of females with PFPS

The scatter plot shows a negative correlation between pain and functional status of females with PFPS i.e. increase in pain during unilateral squat leads to decrease in Kujala score and therefore a reduction in functional status. The value of unstandardized "B" was -2.501 showing that with each point increase in pain on VAS, the functional status is reduced by 2.501 .

\section{Discussion}

Studies have shown reduced hip muscle flexibility and strength to be associated with Patellofemoral pain syndrome.(16)) But despite of all the evidence of reduced hip muscle strength in females with PFPS, the relationships between pain, hip muscle strength and functional status are still unclear. This study was conducted to see if any association exists between Pain, muscle flexibility, muscle strength and functional status of females with patello femoral pain syndrome. Identification of this association would be helpful in making it easier to devise a proper treatment plan that ensures best outcomes and also helps clinicians to develop proper preventive measures for PFPS. In all the previous work done on this issue, the relationship between hip muscle flexibility and weakness with functional status was not well elaborated.

Flexibility of rectus femoris, hamstrings and TFL \& IT band was found to be less in most of the patients with PFPS. Increasing the flexibility of these soft tissues might help in enhancing the functional status of females with PFPS. As shown by a study on increasing IT band flexibility and thereby improving function in patients of PFPS.(17)

The mean strength of hip abductors, lateral rotators and extensors was found to be greater than grade 3 . However, this is an established fact that patients of PFPS have less hip muscle strength.(18) A good averagestrength of hip muscles 


\section{International Journal of Science and Research (IJSR) \\ ISSN (Online): 2319-7064 \\ Index Copernicus Value (2013): 6.14 | Impact Factor (2015): 6.391}

in PFPS patients in our study might be due to the fact that most of the patients had developed PFPS less than a year ago.

Pain in unilateral squat was found to be a good predictor of functional status in females with PFPS and explained considerable variance in functional status. The results of prior studies also have shown the same result.(19)

\section{Conclusion}

The study concludes that pain in unilateral squat and strength of hip extensors were better predictors of functional status in females with patellofemoral pain syndrome.Hip muscle flexibility was not a good predictor of functional status.

\section{References}

[1] Nejati P, Forogh B, Moeineddin R, Baradaran HR, Nejati M. Patellofemoral pain syndrome in Iranian female athletes. Acta Med Iran. 2011;49(3):169-72. Epub 2011/06/18.

[2] Waryasz GR, McDermott AY. Patellofemoral pain syndrome (PFPS): a systematic review of anatomy and potential risk factors. Dynamic medicine : DM. 2008;7:9. Epub 2008/06/28.

[3] Lankhorst NE, Bierma-Zeinstra SM, van Middelkoop M. Risk factors for patellofemoral pain syndrome: a systematic review. J Orthop Sports Phys Ther. 2012;42(2):81-94. Epub 2011/10/28.

[4] Pappas E, Wong-Tom WM. Prospective Predictors of Patellofemoral Pain Syndrome: A Systematic Review With Meta-analysis. Sports Health. 2012;4(2):115-20. Epub 2012/09/28.

[5] Halabchi F, Mazaheri R, Seif-Barghi T. Patellofemoral pain syndrome and modifiable intrinsic risk factors; how to assess and address? Asian J Sports Med. 2013;4(2):85-100. Epub 2013/06/27.

[6] Park SK, Stefanyshyn DJ. Greater Q angle may not be a risk factor of patellofemoral pain syndrome. Clin Biomech (Bristol, Avon). 2011;26(4):392-6. Epub 2010/12/24

[7] Molgaard C, Rathleff MS, Simonsen O. Patellofemoral pain syndrome and its association with hip, ankle, and foot function in 16- to 18-year-old high school students: a single-blind case-control study. J Am Podiatr Med Assoc. 2011;101(3):215-22. Epub 2011/05/31.

[8] Aminaka N, Pietrosimone BG, Armstrong CW, Meszaros A, Gribble PA. Patellofemoral pain syndrome alters neuromuscular control and kinetics during stair ambulation. J Electromyogr Kinesiol. 2011;21(4):64551. Epub 2011/04/29.

[9] Song CY, Lin JJ, Jan MH, Lin YF. The role of patellar alignment and tracking in vivo: the potential mechanism of patellofemoral pain syndrome. Phys Ther Sport. 2011;12(3):140-7. Epub 2011/08/02.

[10] Bolgla LA, Boling MC. An update for the conservative management of patellofemoral pain syndrome: a systematic review of the literature from 2000 to 2010. Int J Sports Phys Ther. 2011;6(2):112-25. Epub 2011/06/30.
[11] Chesworth BM, Culham EG, Tata GE, Peat M. Validation of Outcome Measures in Patients WithPatellofemoral Syndrome 1. Journal of Orthopaedic \& Sports Physical Therapy. 1989 Feb;10(8):302-8

[12] Kujala UM, Jaakkola LH, Koskinen SK, Taimela S, Hurme M, Nelimarkka O. Scoring of patellofemoral disorders. Arthroscopy: The Journal of Arthroscopic \& Related Surgery. 1993 Apr 30;9(2):159-63.

[13] Crossley KM, Bennell KL, Cowan SM, Green S. Analysis of outcome measures for persons with patellofemoral pain: which are reliable and valid?. Archives of physical medicine and rehabilitation. 2004 May 31;85(5):815-22.

[14] Kendall FP, McCreary EK, Provance PG, Rodgers MM, Romani WA. Muscles: testing and function with posture and pain.

[15] Reese NB, Bandy WD. Use of an inclinometer to measure flexibility of the iliotibial band using the Ober test and the modified Ober test: differences in magnitude and reliability of measurements. Journal of Orthopaedic \& Sports Physical Therapy. 2003 Jun;33(6):326-30.

[16] Piva SR, Goodnite EA, Childs JD. Strength around the hip and flexibility of soft tissues in individuals with and without patellofemoral pain syndrome. Journal of orthopaedic \& sports physical therapy. 2005;35(12):793-801.

[17] Tyler TF, Nicholas SJ, Mullaney MJ, McHugh MP. The role of hip muscle function in the treatment of patellofemoral pain syndrome. The American journal of sports medicine. 2006;34(4):630-6.

[18] Robinson RL, Nee RJ. Analysis of hip strength in females seeking physical therapy treatment for unilateral patellofemoral pain syndrome. Journal of orthopaedic \& sports physical therapy. 2007;37(5):2328.

[19] Long-Rossi F, Salsich GB. Pain and hip lateral rotator muscle strength contribute to functional status in females with patellofemoral pain. Physiotherapy Research International. 2010;15(1):57-64.

\section{Author Profile}

Hira Jabeen is Senior Lecturer, Azra Naheed Medical College, Department of Physical Therapy, Main Raiwind Road,, Lahore

Muhammad Salman Bashir is Head of Department/ Associate Professor, Department of physical therapy and rehabilitation sciences, FHAS, ICBS, Lahore

Sarah Ehsan is Senior Lecturer, Azra Naheed Medical College, Department of Physical Therapy, Main Raiwind Road,, Lahore 